\title{
Development and Evaluation of a New Technological Way of Engaging Patients and Enhancing Understanding of Drug Tolerability in Early Clinical Development: PROACT
}

\author{
Andrew Hughes · Donal Landers · Hendrik-Tobias Arkenau • Saj Shah • \\ Richard Stephens · Amrik Mahal · Matthew Simmons · Charlotte Lemech • \\ Jennifer Royle
}

Received: December 9, 2015 / Published online: May 11, 2016

(c) The Author(s) 2016. This article is published with open access at Springerlink.com

\section{ABSTRACT}

Introduction: During early clinical testing of a new medication, it is critical to understand and characterise patient tolerability. However, in early clinical studies, it is difficult for patients to contribute directly to the sponsors'

Enhanced content To view enhanced content for this article go to www.medengine.com/Redeem/03C4F0602 CFBD85A.

J. Royle was an employee of AstraZeneca at the time this research was conducted.

\section{A. Hughes}

Manchester Cancer Research Centre, University of

Manchester, Manchester, UK

D. Landers · S. Shah $\cdot$ A. Mahal · J. Royle

AstraZeneca, Cambridge, UK

Hendrik-TobiasArkenau · M. Simmons · C. Lemech Sarah Cannon Research Institute, London, UK

Hendrik-TobiasArkenau · C. Lemech

University College London, London, UK

\section{R. Stephens}

Consumer Forum, National Cancer Research

Institute, London, UK

Present Address:

J. Royle $(\square)$

Cancer Research UK Manchester Institute, The

University of Manchester, Manchester, UK

e-mail: Jenny.Royle@cruk.manchester.ac.uk understanding of a new compound. Patient reported opinions about clinical tolerability (PROACT) provides a new, simple and innovative way in which patients can collaborate using an application downloaded to a mobile computer or smartphone.

Methods: PROACT was designed with special consideration given to patient confidentiality, patient engagement and data security. A pilot study was conducted to investigate patient uptake of PROACT and to characterize clinical trial information it captured. Patients recruited to Phase I oncology trials at a UK center were eligible to participate but were required to have a tablet computer or smartphone. Patients used PROACT to upload audio/video messages that became available instantly to their clinical team, who were able to reply to the patient within PROACT. The patient's message was also analyzed, personally-identifiable information removed and anonymized information then made available to the sponsor in an analytics module for decision-making. In parallel, a patient focus group was engaged to provide feedback on communication needs during early clinical trials and the PROACT concept. 
Results: Of the 16 patients informed of PROACT, 8 had a smart device and consented to take part. Use of PROACT varied and all messages volunteered were relevant and informative for drug development. Topics disclosed included tolerability impacts, study design, and drug formulation. Alignment with the clinical study data provided a richer understanding of tolerability and treatment consequences. This information was available to be shared among the clinical team and the sponsor, to improve patient support and experience. Patient forum feedback endorsed the concept and provided further information to enhance the application.

Conclusion: Overall, PROACT achieved proof of concept in this small pilot study and delivered a secure end-to-end system that protected patient privacy and provided preliminary insight into patient experiences beyond the usual clinical trial data set. The use of mobile devices to interact actively with participants in clinical trials may be a new way of engaging and empowering patients. Further validation of this technology in larger patient cohorts is ongoing.

Funding: AstraZeneca.

Keywords: Oncology; Phase I; Patient feedback; PROACT; Safety; Tolerability

\section{INTRODUCTION}

Pharmaceutical innovation is based on cutting edge science with the ultimate aim of improving patients' lives. However, the path from innovation through development is complex and highly regulated [1] with well-defined but proscriptive technical requirements for the conduct of clinical trials [2]. Furthermore, patient outcomes are also dependent on compliance [3], and those who do not engage, and therefore, do not comply are likely to derive lesser benefit. It is no longer acceptable to rely solely on the scientific output of the clinical study when the patient's experience of the drug and choices made may be of equal importance, both to the sponsor and to the patient.

The principal objective in an open-label Phase I oncology study, is to understand safety, tolerability and other aspects of the drug profile, and thus help define a recommended dose for further investigation [4]. A major component in determining this is deciding which dosing regimen is best tolerated by patients for the duration of study treatment. This is not easy to address with the clinical dataset alone, which is entered by the clinical teams in the clinical trial site, and categorical in the nature of the data collection, particularly recording of adverse events. For example, there may be significant resolution in the severity or distribution of an event which still remains within the same severity category, e.g., mild. As the patients themselves cannot ordinarily share their experiences directly with a clinical study sponsor, it is generally the information in the case report form [5] that is solely relied upon for drug development decisions. In this case, this gives the impression of constancy when in fact, there may have been, to the patient at least, a significant reduction in the treatment burden experienced. Incorporating patient-driven insight, that is shared directly with both the medical teams and study sponsors, into this understanding is likely to provide a step-change in interpretation capability and to result in more informed decisions [6, 7], particularly for those adverse events that are not categorized as being serious.

Laws and regulations governing clinical trials and data privacy are there to protect patients 
not to stifle innovation and productivity. Yet, industry, in general, has found it a challenge to embrace technology within this clinical trial environment. One of the main concerns among clinical trial researchers about technology is the risk that the integrity of the clinical study gets compromised by introducing technology aimed at giving some level of control back to the patient. PROACT (patient reported opinions about clinical tolerability) is an application that is designed to empower patients participating in early clinical studies by giving them a way to directly contribute to drug development on their terms. Patients are at complete liberty to decide whether they want to participate, what information they volunteer, and when they do so, giving them the unique opportunity to contribute as a partner in drug development. In designing this approach, the PROACT team embraced the principles of relevant laws and regulations as part of the solution, not the problem. They used privacy by design and managed to balance the need to maintain the integrity of the clinical trial process so that the clinical trial itself remained objective, unbiased, and uncompromised in its scientific integrity. PROACT breaks through the traditional pharma concept of a 'communication gap' between the patients in studies and the researchers developing the drug.

As the PROACT approach is so radically different to traditionally accepted methodology, and shifts the information flow from the sponsor and/or clinical teams, to the patients who drive and choose content, testing this system for the first time brought with it a high level of uncertainty. Therefore, we developed a pilot system, and conducted a pilot study that was limited in scale so that the utility of PROACT could be explored in the clinical oncology setting, without inadvertently increasing risks in other areas. Furthermore, we conducted in parallel a patient forum meeting to enhance our learning and implementation of the system by gathering information on communication needs within early clinical trials and pulling this into the PROACT approach. It is the learning gathered during the pilot and the correlating patient focus group meeting that is presented in this report.

\section{METHODS}

\section{PROACT Design and Development}

PROACT was a password-protected mobile application developed with ease of use and security in mind. It had a help function that contains a video on how to use the application, and a link to a technical support team for help with password resets and any technical issues. The application allowed patients to record messages (audio/video) and for these to be streamed to a server and made instantly viewable to the patient's onsite clinical team who were then able to respond appropriately. Patients received a return video/audio message in a chronologically arranged 'message list' that they were able to view at will.

After a delay of 32 days, the patient's message was sent through to a small group of independent analysts. This delay was to ensure that any adverse event information was discussed between the patient and their onsite clinical team and that all relevant updates were entered into the clinical study database for reporting as per the clinical study protocol. The analysts removed personally-identifiable information while capturing the patient message which was then relayed to the sponsor's study team for further analysis (see Fig. 1). 


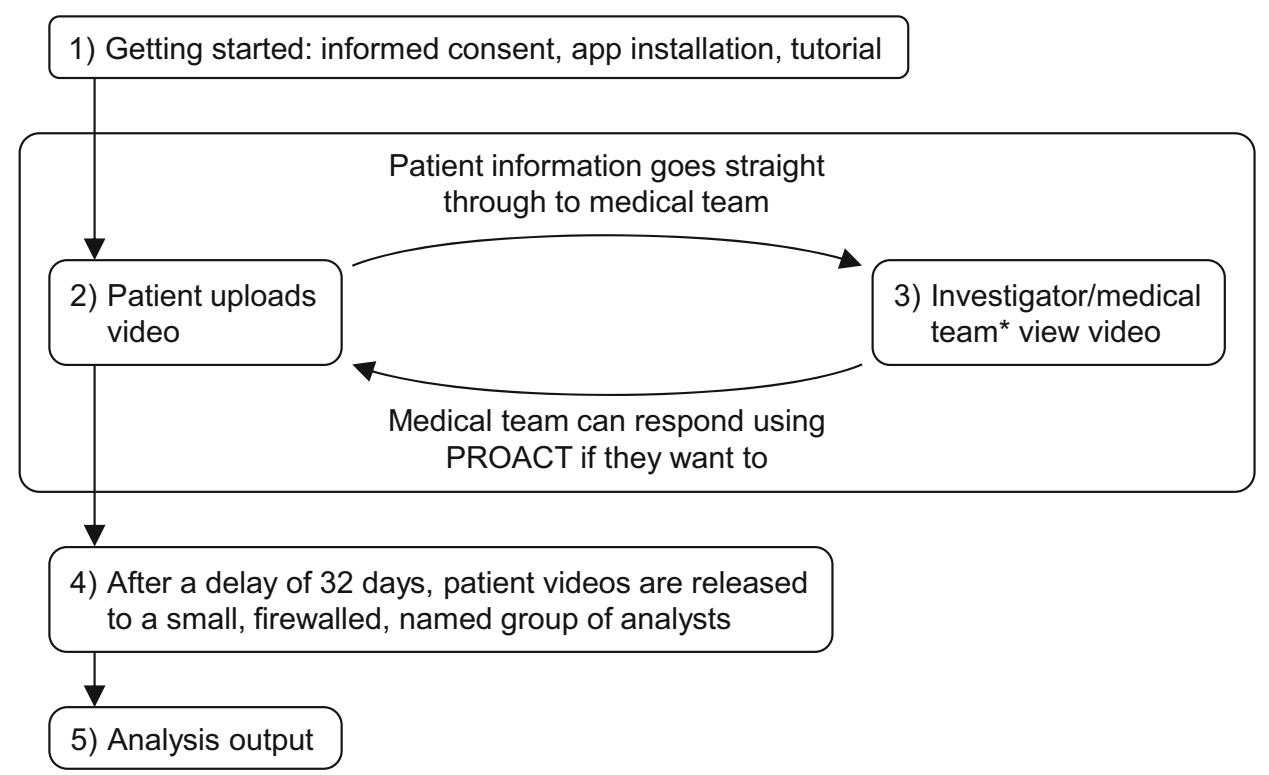

*Medical team: a small group of pre-defined medical team members that the investigator wished to have access to this information (eg, research nurse). Each had a secure log-in to PROACT, but all members of this team had access to the same account to facilitate sharing of information and collaboration between medical staff assigned to that patient.

Fig. 1 Information flow using PROACT

Patients and medical team members had the ability to trigger deletion of any of their own videos recorded and uploaded to the PROACT system by pressing the 'delete' button within the application. This triggered total deletion of that video and associated information (but not analyzed/anonymized data).

\section{Data Storage and Protection}

Rigorous controls were implemented to ensure data protection and patient confidentiality. All connections to the PROACT application were secured via https using a digital certificate. Within the PROACT environment, encrypted data were hosted by the Safe Harbor Certified Provider (Rackspace) in accordance with national and international standards on data protection [8-10]. User access to decrypted data was granted at different levels dependent on role (patient, investigator, administrator, sponsor).

\section{Pilot Study Objectives}

The pilot study was conducted using a clinical protocol, which had been developed, written and submitted to an Ethics Committee for approval. The objectives were to investigate the uptake and use of PROACT when made available to patients undergoing treatment for cancer in a clinical trial setting and to characterize the information that patients volunteered. The principal focus in this respect was to understand the technicalities and practicalities of the application. The study was also designed to preliminarily assess the potential benefit of receiving direct feedback from patients for the sponsor, and to explore analysis methods that would convert the information into an interpretable dataset while also protecting patient privacy. The objectives were developed with the long-term aim of PROACT being used in a way that fits with patients' day-to-day lives. 
Study Design, Participants, and Data

\section{Collection}

Patients aged $\geq 18$ years recruited to Phase I AstraZeneca (the sponsor) oncology studies at the Sarah Cannon Research Institute, London, UK were eligible to participate. Patients were also required to have a "smart" phone or tablet computer that they were willing to use (without reimbursement) for the transmission of audio/ video recordings. Provision of informed written consent was also required.

Following enrolment, patients downloaded the PROACT application to their "smart" phone or computer tablet and were instructed on its technical use. Beyond technical advice, patients were provided minimal guidance on use of the system. This allowed the sponsor to gather insight into the nature and level of support that PROACT use may require in the future, albeit from a small sample of patients already comfortable with using mobile devices. Patients' messages were created, transmitted and handled as described previously.

\section{Data Analysis}

Data evaluation focused on identifying contextual key words and topics, using manual text analysis of transcribed data. Patient-identifiable data was removed while capturing the message a patient wanted to convey in an interpretable dataset. To enable this, a hierarchical dictionary and flexible coding database were created to allow for aggregation of data, while linking back to the patient's own words (see Fig. 2). The database comprised a series of entries, each comprising a coded patient identity, date of communication, data category (e.g., safety, efficacy, convenience), data group (e.g., system affected), topic (e.g., specific treatment burden), the patient quote, status of the communicated event/issue, and response/ outcome. Also, a simple color scale was included to visualize the impact of the reported topic upon that patient's wellbeing or daily activities [green, minimal impact on quality of life (also used to describe if an event had resolved or was improving); amber, moderate impact on quality of life, able to generally continue daily living activities with some limitation (also used to describe an ongoing event); red, significant impact on quality of life (also used to describe a new or worsening event)]. The nature and content of the messages, therefore, drove the development of the dictionary and coding database in an iterative process. These outputs were then assessed to see whether they were able to capture the core elements of the patient contribution in a format that would be useful for contributing to drug programme decisions.

\section{Ethical Approval}

The study received Ethical Research Committee approval (with positive comments regarding steps taken to ensure patient confidentiality and data protection) and it was conducted in accordance with applicable regulatory requirements and other relevant guidelines, such as patient data privacy requirements previously described [8-10].

\section{Patient Forum}

In parallel with the pilot study, we conducted an interactive meeting with patient and carer representatives of the National Cancer Research Institute's Consumer Forum (in the UK). Participants in a patient focus group were invited to provide insights into the needs of cancer patients during treatment with a focus 


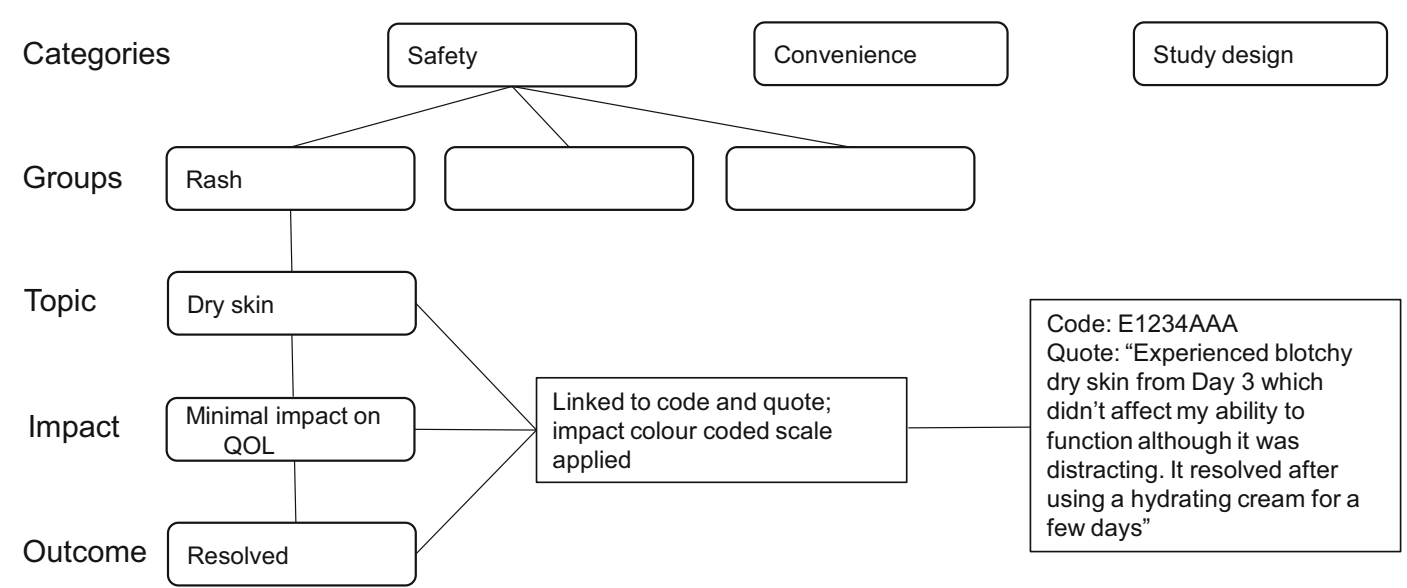

Fig. 2 Hierarchical dictionary and flexible coding database; terminology tree example. QOL quality of life

on communications in the clinical trial environment. Open forum discussions initially concentrated on three main areas: (1) current clinical practice, (2) patient needs, and (3) carer needs and contributions. The first half of the discussion focused on people's experiences and opinions before the PROACT concept was unveiled (to mitigate against any bias that proposing PROACT may have introduced). The second half of the session focused on discussion and input into PROACT itself: the concept, approach, use, and guidance. Information gathered was used to assess whether any improvements could be made to the use of PROACT during the pilot study, and as well as aiding development of an enhanced version if further evaluation of the application was deemed appropriate.

\section{RESULTS}

\section{Patients}

Of the 16 patients informed of the PROACT initiative (12 male, 4 female), 8 patients already possessed and used a computer tablet or smartphone and consented to take part. Participating patients were marginally younger (mean age 61 years; range 45-68 years) than those ineligible to participate through lack of access to mobile technology (mean age 67 years; range $51-80$ years).

\section{Usage and Information Shared}

The use of PROACT varied between individuals, with some patients using the application many times a week and others rarely or not at all (the latter stated that they did not feel that they had much or anything to share). All messages volunteered by patients were very relevant and informative for drug development. Topics covered included the impact of safety and tolerability, relay of self-monitored results (e.g., blood pressure), feedback on study design, and information relating to the formulation of the medications (see Table 1). Patient feedback on the PROACT approach itself was also positive, highlighting the patient's role in drug development (e.g., "It is gratifying to know that I have a role in the development of the drug. To believe that this may help others in the future is a real reward too.").

Patient feedback via PROACT provided an extra level of insight. Alignment with the clinical study data provided a richer 
Table 1 Examples of patient feedback received by topic

\begin{tabular}{ll}
\hline Topic & Example feedback \\
\hline Safety & Patient was monitoring own blood pressure and sending results \\
back to medical team in between visits \\
Example quotes include: \\
"I spent Sunday in bed shaking off a headache and feeling unwell” \\
"I'm not doing an awful lot because of the fatigue that is really quite, \\
quite overwhelming" \\
"The taste of the pills is unpleasant even though they are in the mouth \\
for only a couple of seconds, ... If it was possible to mask the taste \\
of the pills that might help" \\
"A 12 h interval between taking the medicine which requires a $3 \mathrm{~h}$ fast, \\
2 before and 1 after, is inconvenient as say, 0700 h [morning administration] \\
and $1900 \mathrm{~h}$ [evening administration] ... is not a good time in the evening to \\
fast if you are trying to live a normal life" \\
"I have been fairly tired as this is second day after chemo and the fairly \\
demanding day of giving blood for the trial”
\end{tabular}

Table 2 Example comparison of clinical and PROACT data relating to the topic

\begin{tabular}{ll}
\hline Data source & Information recorded/feedback provided \\
\hline Clinical study database & MedDRA term 'Rash', CTCAE Grade 1, ongoing \\
PROACT & "The rash is gone from my face and is greatly reduced on my chest" \\
& "It is also quite unpleasant to expose my skin to hot \\
& water-I don't enjoy a shower or a bath" \\
"My skin continues to be dry but is responding to & stuff called Bach's rescue cream and is not a problem" \\
\hline
\end{tabular}

MedDRA medical dictionary for regulatory activities, CTCAE common terminology criteria for adverse events

understanding of tolerability, the impact on patients and the overall consequences (see Table 2).

\section{Data Analysis}

The coded database was visualized in a series of graphs to explore potential signals (Fig. 3). All data points were supported with the patient's own words and these were readily accessible so that the patient's intended message could be seen by the clinical team.

This ability to work with both aggregated individual data provided efficient interpretation and alignment of patient contributions. Thus, the study sponsor was able to (1) spot trends, such as the most frequently talked about topics and the temporal emergence of topics, (2) identify the aspects of study treatment and/or study conduct having most impact on patients, 
(a)
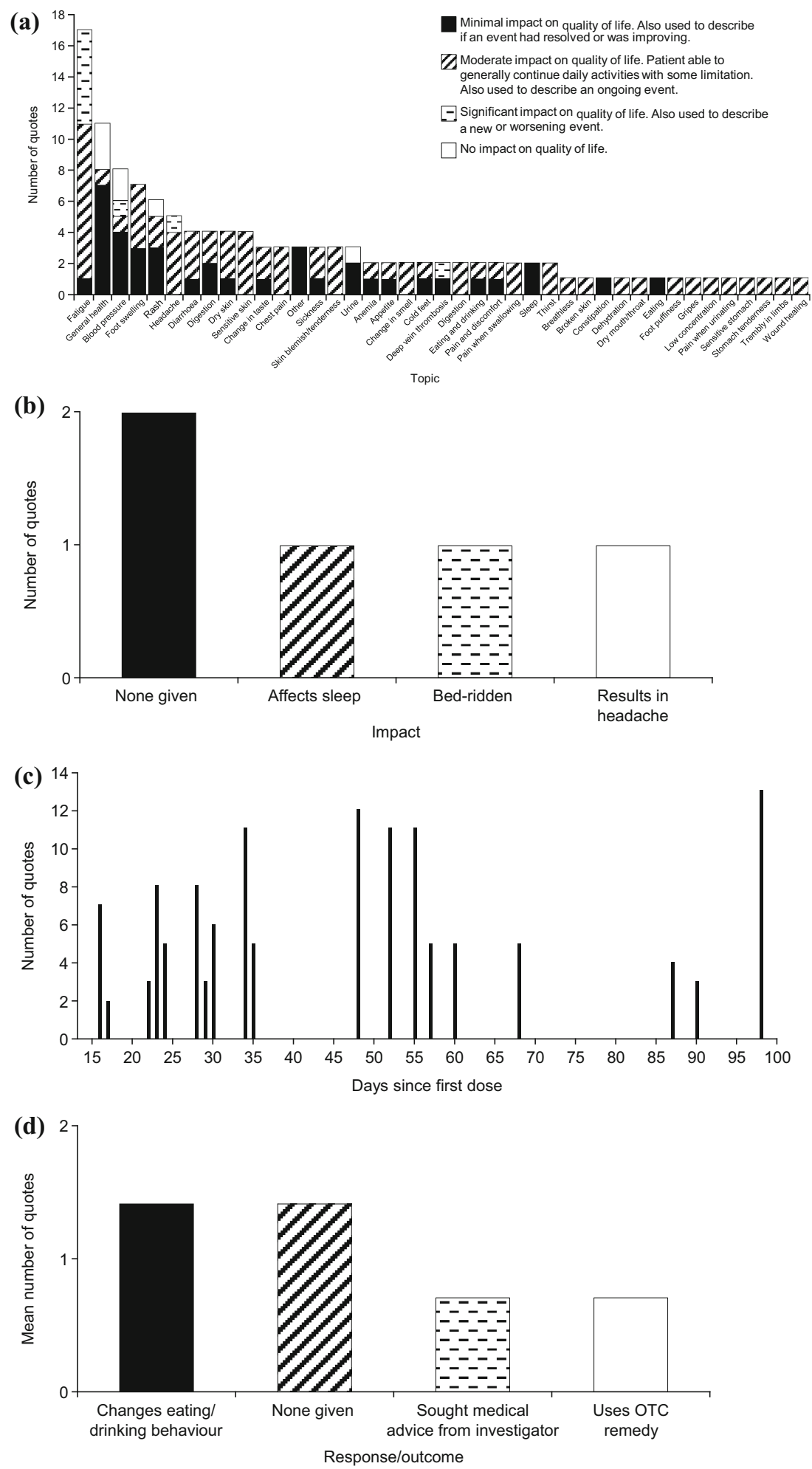

Fig. 3 Analysis and graphical representation of feedback through PROACT. a Total number of quotes by topic. b Reported impact of condition/medication on health. c Total number of quotes over time. $\mathbf{d}$ Response/outcome. OTC over-the-counter 
and (3) identify management techniques that provide the greatest benefit for patients, including some techniques being developed by patients themselves.

\section{Patient Forum Feedback}

A total of five patients participated in open discussions regarding communication in early clinical trials and provided evaluation of the PROACT concept. General feedback on communication within early clinical trials showed that there is a need for greater communication involving patients, carers and health care providers, with a focus on patient experience to build empathy, provide reassurance, ensure transparency and to document more patient-orientated information (see Table 3). In the following session, patients were briefly told of the PROACT concept and were asked to discuss its potential features and usage (see Table 4). The group generally agreed that the concept was advantageous and that having a means to send messages between site visits would provide extra insight into the patient experience. Patients considered that being able to send messages ad hoc and at scheduled times to both be beneficial, and they considered an option to receive messages, particularly relating to study status, a key attribute. However, they considered that uptake and usage would depend on ease of use, while security features should be pitched at an appropriate level so as to not be off putting.

\section{DISCUSSION}

This study was intended to provide feedback on the utility of PROACT in a small cohort of patients in early oncology clinical trials. Mobile technology has been previously shown to improve clinical outcomes when an interactive application was used by patients and health care providers outside a clinical trial [11]. However, the PROACT interactive application is the first to our knowledge to have been used to give patients a way to contribute to drug understanding on their own terms within a clinical trial. Indeed, this pilot study demonstrated that PROACT is a valid approach to engage and empower patients in early oncology clinical trials and to gain further insight into their experience beyond that captured in the traditional clinical data set. It facilitated communication between patients, the clinical team, and the sponsor, on specific topics such as, safety, dosage administration, and study design, while also providing added and complementary information on tolerability.

The development of PROACT has required successful navigation through the complex regulatory, ethical, and legal landscape of the pharmaceutical industry and regulatory environments. The outcome was a system and process that ensured patient confidentiality and security of data. PROACT also demonstrated that patient communications could be captured and interpreted in a useful format by implementing a hierarchical dictionary and flexible coding database. Bringing these elements together, this small study showed that there are significant potential benefits for patients, the clinical team, and the sponsor. Patients receive an easy-to-use video communication application and a way of linking to a key 'anchor' person at the clinical trial site. The onsite clinical team is able to see and review the patients simply and quickly between visits. As patients' insight is also aggregated for the overall study population, they also receive a better understanding of drug tolerability, thereby gaining a direct understanding from patients across the whole 
Table 3 Patient feedback on participation in clinical trials; aspects related to communication

\begin{tabular}{|c|c|c|}
\hline Topic & Finding & Comment/summary \\
\hline \multirow[t]{3}{*}{$\begin{array}{l}\text { Current } \\
\text { clinical } \\
\text { practice }\end{array}$} & $\begin{array}{l}\text { Feedback reporting does not always capture the right } \\
\text { information (e.g., patient-reported outcomes } \\
\text { questionnaires) }\end{array}$ & $\begin{array}{l}\text { "... there was no opportunity to get any sort of personalised } \\
\text { data"... "There was no emotional-type stuff, to convey how } \\
\text { you were feeling" }\end{array}$ \\
\hline & \multirow[t]{2}{*}{ There is a communication gap in overall health provision } & $\begin{array}{l}\text { Although there are communication channels to the GP } \\
\text { (dependent on whether the site investigator approves), } \\
\text { A\&E, and clinical study site there is still an unmet need }\end{array}$ \\
\hline & & $\begin{array}{l}\text { 'Clinical site: emergency } 24 \text { help number, but what if it's not } \\
\text { an emergency just something that's bothersome? GP surgery: } \\
\text { can't get an appointment. A\&E: only if you're 'dying'. There } \\
\text { is a need for a channel where you can communicate when } \\
\text { you need to and you know that the medical team will pick it } \\
\text { up soon and you'll get a response, but that you're not } \\
\text { interfering" }\end{array}$ \\
\hline \multirow[t]{3}{*}{$\begin{array}{l}\text { Patient } \\
\text { needs }\end{array}$} & There is a need for a knowledgeable "anchor" person & $\begin{array}{l}\text { An anchor person should be available to answer questions and } \\
\text { provide reassurance-not at set appointments. The response } \\
\text { doesn't have to be immediate but it does need to be } \\
\text { provided within polite/reasonable timelines }\end{array}$ \\
\hline & There is a need for open-ended patient feedback & $\begin{array}{l}\text { Patients want to tell people some things that they never get } \\
\text { asked. Simple things such as 'what do you think of the shape } \\
\text { of the tablets'. No one ever asks, but sometimes it might be } \\
\text { something that is quite difficult to deal with. To just be able } \\
\text { to tell someone who can potentially do something about it }\end{array}$ \\
\hline & $\begin{array}{l}\text { There is a need to provide opportunities to build empathy } \\
\text { and to share information }\end{array}$ & $\begin{array}{l}\text { Patients need to feel valued and that they are helping to "build } \\
\text { something" - this can be motivational and provides a } \\
\text { purpose for participation. Patients also want to know what } \\
\text { is "going on" }\end{array}$ \\
\hline Carer needs & Carers should have the opportunity to communicate too & $\begin{array}{l}\text { Carers can raise issues that the patient may not perceive, but } \\
\text { there should be separate communication channels for carers } \\
\text { and patients to ensure patient privacy and to provide a route } \\
\text { of communication back to the patient if appropriate. Carers } \\
\text { may also communicate on behalf of a patient where the } \\
\text { patient is reluctant or has inhibitions about communicating } \\
\text { their true experience }\end{array}$ \\
\hline
\end{tabular}

$A \& E$ accident and emergency, $G P$ general practitioner

study and in a non-clinical setting, i.e., at home. This enables better understanding and better patient management of tolerability issues during an ongoing clinical study and builds a 'patient' knowledge base, which may be used for subsequent related studies. Also, the sponsor receives broad insight into the impact the drug is having on patients that includes information related to tolerability, formulation, convenience, and study conduct. This enables assessment of the recommended dose on a comprehensive dataset generated from both the clinical report form and direct patient feedback.
Limitations of this pilot study include the small sample size, the iterative process that led to the development of the coded database (no saturation criteria were used for feedback topics), use of an early version of the application, and the low uptake rate due to patients not possessing the appropriate technology. Of note, feedback from patients on the use of the application itself was positive, but in spite of this, it became apparent that patients, in general, were not aware of the value their contribution could make. Patients sometimes did not share insight in the pilot 
Table 4 Patient feedback on the PROACT concept

\begin{tabular}{|c|c|c|}
\hline Topic & Finding & Comment/summary \\
\hline \multirow[t]{3}{*}{$\begin{array}{l}\text { Inclusion } \\
\text { within a } \\
\text { study }\end{array}$} & $\begin{array}{l}\text { Having a way to send messages between } \\
\text { visits may provide extra insight }\end{array}$ & $\begin{array}{l}\text { "The problem we have at the moment is that the only way an } \\
\text { issue [such as the way you feel] is captured is when we go back } \\
\text { to see the doctor after } 48 \mathrm{~h} \text {, after } 1 \text { week, after } 2 \text { weeks, } \\
\text { whenever the next cycle is due. Which means [by that time] } \\
\text { (A) we've probably forgotten how bad we felt, (B) we've } \\
\text { probably forgotten some aspects, and (C) we may be more } \\
\text { focused on ... getting the next dose" }\end{array}$ \\
\hline & $\begin{array}{l}\text { Patients may benefit from ability to record } \\
\text { both ad hoc and scheduled messages }\end{array}$ & $\begin{array}{l}\text { "I can see it as a tool for contacting doctors. I think it's brilliant. } \\
\text { But in terms of a tool for contributing to a trial on a regular } \\
\text { basis that might be hard to achieve" }\end{array}$ \\
\hline & & $\begin{array}{l}\text { "On the other hand, it could genuinely be quite helpful in itself, } \\
\text { but also quite motivational. We take part in trials-yes of } \\
\text { course we want the benefit for ourselves-but the other reason } \\
\text { we do it is to help other people ..." }\end{array}$ \\
\hline $\begin{array}{l}\text { Study } \\
\text { updates }\end{array}$ & $\begin{array}{l}\text { Study updates are considered to be key } \\
\text { elements of communication }\end{array}$ & $\begin{array}{l}\text { Content: basic updates on study design and progress. } \\
\text { Recruitment? Is the drug working in anyone? Emerging side } \\
\text { effects? Whatever is possible, given that patients understand } \\
\text { not everything is possible. Related trials. What have we learnt? } \\
\text { What's happening next? }\end{array}$ \\
\hline $\begin{array}{l}\text { User } \\
\text { interface }\end{array}$ & $\begin{array}{l}\text { Ease of use is considered integral } \\
\text { to uptake }\end{array}$ & $\begin{array}{l}\text { User experience of technology is integral to whether people } \\
\text { communicate and become involved. Button to switch on/off } \\
\text { camera (i.e., option to record audio or video that is clearly } \\
\text { delineated). No strong desire for typing, if anything, the } \\
\text { opposite. Request for filtering/search facilities. Request for } \\
\text { emoticons on video as optional-patients setting sentiment } \\
\text { that they had at the time themselves. Pop up reminders for } \\
\text { schedule. Notification for delay in response }\end{array}$ \\
\hline $\begin{array}{l}\text { Security } \\
\text { features }\end{array}$ & $\begin{array}{l}\text { Security features need to be appropriate } \\
\text { but not off-putting }\end{array}$ & $\begin{array}{l}\text { Remembering pin codes can be a problem for some patients. } \\
\text { There is a need to consider the security of storing information } \\
\text { on the device versus instant streaming. Instant-stream } \\
\text { messaging may be inconvenient, especially if it does not work } \\
\text { offline }\end{array}$ \\
\hline
\end{tabular}

study simply because they did not think that anyone would want to know about it. This is a hurdle that needs to be overcome, so that decisions in clinical management and drug development can be based on a broad and rich understanding of patient experiences and needs. Additional guidance will be developed and made available for both patients and medical teams in future. This will include the points that have been learned from the pilot study, topics of interest, the usefulness and value of the information, and imbedding PROACT within standard clinical study communication channels. Additional insight 
from the patient forum will be used to develop an enhanced version of the PROACT application that will also be made available for download to laptop/desktop computers. It is intended to provide more information on the use of the application and information relating to the study medication together with comprehensive management guidelines (including practical tips from previous patient experiences when taking the drug). In the wider context, it is intended that PROACT be incorporated as an element in the drug development process, contributing to bioinformatics and providing patients with personalized data feedback.

\section{CONCLUSIONS}

In summary, PROACT achieved initial proof of concept and delivered a secure end-to-end system that protected patient privacy while providing preliminary patient insight into clinical trial participation. In this respect, it appears to provide a new way of engaging and empowering patients with the potential to change the communication paradigm for an ongoing clinical trial. As previous studies have shown, engaged patients who have the skills, ability, and willingness to help manage their own health have better health outcomes at lower cost to the health system [12]. Further validation of this technology in larger patient cohorts using an enhanced application is ongoing.

\section{ACKNOWLEDGMENTS}

We thank all the patients who took part in the study, colleagues within the Sarah Cannon Research Institute UK site who cared for these patients, and Bartley O'Connor of Sqeeler
Limited for the IT platform used during this pilot study. We also thank David Peters of Sequoia Medical Communications Ltd, who provided medical writing assistance funded by AstraZeneca. This study was sponsored by AstraZeneca. The article processing charges and open access fee for this publication were funded by AstraZeneca. All named authors meet the International Committee of Medical Journal Editors (ICMJE) criteria for authorship for this manuscript, take responsibility for the integrity of the work as a whole, and have given final approval for the version to be published.

Disclosures. All research was funded and supported by AstraZeneca. At the time the research was conducted, Andrew Hughes, Donal Landers, Saj Shah, Amrik Mahal, and Jennifer Royle were all employed by AstraZeneca. Hendrik-Tobias Arkenau, Matthew Simmons, and Charlotte Lemech were employed by Sarah Cannon Research Institute, London, UK. Hendrik-Tobias Arkenau, and Charlotte Lemech were also employed by University College London, London, UK. Richard Stephens is a patient advocate who received consultancy fees from AstraZeneca for his time.

Compliance with Ethics Guidelines. The study received Ethical Research Committee approval (with positive comments regarding steps taken to ensure patient confidentiality and data protection) and it was conducted in accordance with applicable regulatory requirements and other relevant guidelines, such as patient data privacy requirements previously described [8-10].

Open Access. This article is distributed under the terms of the Creative Commons Attribution-NonCommercial 4.0 International License (http://creativecommons.org/licenses/ 
by-nc/4.0/), which permits any noncommercial use, distribution, and reproduction in any medium, provided you give appropriate credit to the original author(s) and the source, provide a link to the Creative Commons license, and indicate if changes were made.

\section{REFERENCES}

1. Rago L, Santoso B. Drug regulation: history, present and future. In: van Boxtel CJ, Santoso B, Edwards IR, editors. Drug benefits and risks: international textbook of clinical pharmacology, 2nd ed. IOS Press and Uppsala Monitoring Centre; 2008.

2. International Conference on Harmonization of Technical Requirements for Registration of Pharmaceuticals for Human Use 2015. http:// www.ich.org/home. Accessed Dec 7, 2015.

3. Osterberg L, Blaschke T. Adherence to medication. N Engl J Med. 2005;353(5):487-97.

4. Miller FG, Joffe S. Benefit in phase 1 oncology trials: therapeutic misconception or reasonable treatment option? Clin Trials. 2008;5(6):617-23.

5. Bellary S, Krishnankutty B, Latha MS. Basics of case report form design in clinical research. Perspect Clin Res. 2014;5(4):159-66.
6. Basch E, Jia X, Heller G, et al. Adverse symptom event reporting by patients vs clinicians: relationships with clinical outcomes. J Natl Cancer Inst. 2009;101(23):1624-32.

7. Di Maio M, Gallo C, Leighl NB, et al. Symptomatic toxicities experienced during anticancer treatment: agreement between patient and physician reporting in three randomized trials. J Clin Oncol. 2015;33(8):910-5.

8. Standards for privacy of individually identifiable health information DHHS [45 CFR Parts 160, $162 \&$ 164 (HIPAA)].

9. Privacy and Electronic Communication EU directive 2002/58/EC.

10. Framework Data Protection Directive 95/46/EC.

11. Halpin DM, Laing-Morton $\mathrm{T}$, Spedding $\mathrm{S}$, et al. A randomised controlled trial of the effect of automated interactive calling combined with a health risk forecast on frequency and severity of exacerbations of COPD assessed clinically and using EXACT PRO. Prim Care Respir J. 2011;20:324-31.

12. KPMG. Creating new value with patients, carers and communities 2015. http://www.kpmg.com/Global/ en/IssuesAndInsights/ArticlesPublications. Accessed Dec 7, 2015. 